\title{
COMPACT ELECTRON ACCELERATOR FOR RADIATION TECHNOLOGIES
}

\author{
S. Korenev, STERIS CORPORATION, STERIS Isomedix Services, \\ Libertyville, IL 60048, USA
}

\section{Abstract}

The concept of pulsed compact electron accelerator for radiation technologies is considered in the report. The main parameters of this accelerator are next: kinetic energy is $100-700 \mathrm{keV}$, pulse duration is $50-1000 \mathrm{nsec}$, beam current is $100-1000 \mathrm{~A}$, repetition is $1-50 \mathrm{~Hz}$, max dimensions of beam are $1 \mathrm{x} 1 \mathrm{mxm}$..

\section{INTRODUCTION}

The development of radiation technologies (curing of composite materials, sterilisation of packaging materials, surface modification of materials, food irradiation and other) on the basis of electron beam made big progress in the understanding of main requirements on the electron accelerators [1]. The main principal question to accelerator for radiation technologies is regulation of kinetic energy and electron beam power [2]. The alternative methods of treatment for materials on the basis of plasma, UV, ozone and other allow to have only surface treatment of materials. The electron beam permits to have depth method of treatment of materials. But large spectra of irradiated products with different physical and chemical properties leads to design of electron accelerators with regulation of kinetic energy and beam power and consider the problem of irradiation in the combine with conveyer systems.

The analysis of different electron accelerators together with conditions for irradiation shows that the pulsed accelerators can be useful for first step of development of radiation technologies including research topics.

The variant of compact pulsed electron accelerator with regulation of kinetic energy and power beam is considered in the report.

\section{MAIN PROCESSES OF DISSIPATION FOR BEAM ENERGYIN THE IRRADIATED PRODUCT}

The general concept for interaction of electron beams with irradiated product for radiation technologies is dissipation of beam energy in this product.

The main demand for radiation technologies is homogeneous distribution of absorbed doses in the irradiated product.

The typical distribution of absorbed doses in irradiated product is given on Figure1 for one side's irradiation.
The value of average absorbed doses Daveon the level of $20 \%$ is [3]:

$$
\text { Dave }=[\mathrm{Dmax}+\mathrm{Dmin}] / 2,
$$

where: Dmax is max absorbed dose, Dmin is min absorbed doses, see Figure1.

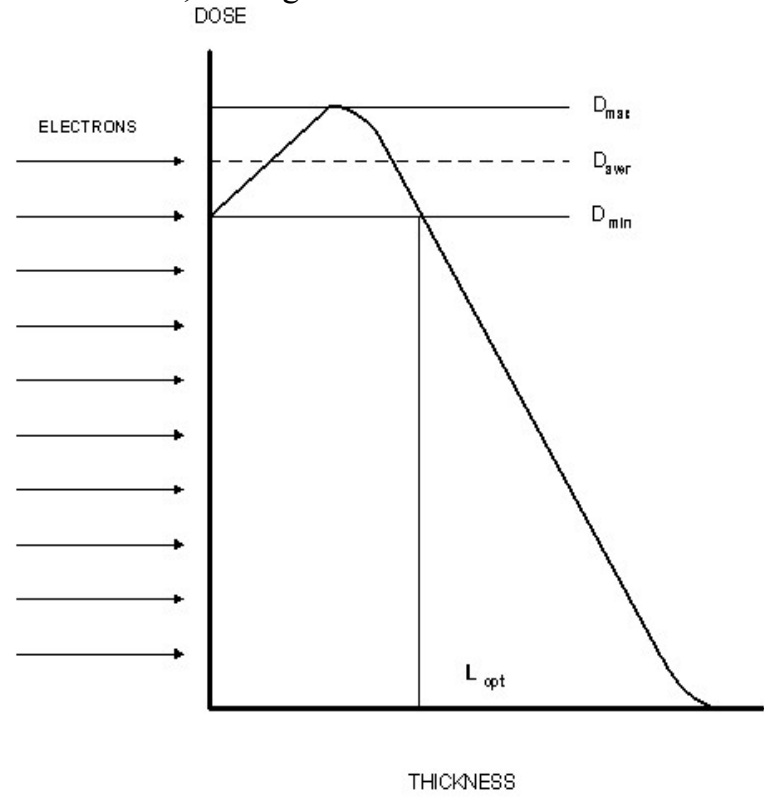

Figure 1.

The optimal thickness Lopt of an irradiated product is determined by this distribution. The thickness of irradiated product is $0.05-2.0 \mathrm{~mm}$ for many materials with density to till $2 \mathrm{~g} / \mathrm{cc}$ and kinetic energy of electrons $100-700 \mathrm{keV}$. The simple calculations of absorbed doses for materials with same thickness shows the level of doses about $50-200 \mathrm{kGy} /$ pulse in case of using of pulsed high current accelerators. It allows to use pulsed high current electron accelerators in the development of radiation technologies. The average level of absorbed doses for many radiation technologies is about 10-150 kGy.

The other very important aspect of advantages of pulsed irradiation is short time of irradiation. The large absorbed doses leads to heating processes and non-control chemical reactions. It is very important for composition materials. The pulsed mode of accelerators allows the adiabatic irradiation. It is more simple in comparison with scanning DC and RF Linacs. The time of irradiation is main variable parameter for many industrial electron 
accelerators. This time of irradiation (dissipation of beam energy) can be determines the thermal characteristics. The parameter for characterization of thermal properties of irradiated materials is local thermal time constant, which can be calculate by formula [4]:

$$
\mathrm{T}(\mathrm{p})=2 \mathrm{~h} 2 \mathrm{rC} / 1
$$

Where: $\mathrm{h}$ is depth of penetration for electron beam in irradiated sample; r,C,l is density, heat capacity and heat conductivity of irradiated materials.

According analysis of thermal distribution for many materials the time or irradiation for pulsed beam with duration aboutb50 - $1000 \mathrm{nsec}$ is smaller in comparison with local thermal time constant ( from ten to till hundred microseconds). This case we can consider as a adiabatic conditions and temperature of the product will have step distribution.

It is very important for multi-component materials with complex stoichiometry relationship.

\section{CONCEPT OF PULSED ELECTRON ACCELERATOR FOR RADAITION TECHNOLOGIES}

The main concept of pulsed electron accelerator for radiation technologies consists in the next:

- receiving of high absorbed doses in the small thickness of sample or product.

- adiabatic conditions of irradiation of samples or products;

- regulation of kinetic energy of electrons;

- regulation of beam power;

- large dimensions of electron beams;

- using of vacuum insulation for high voltage generator;

- $\quad$ simple cooling system for output window;

- using the output foil windows with protective coatings;

- on-line diagnostics of parameters for electron beams;

- low cost of accelerator;

- simple operation and reliable.

The compact pulsed electron accelerator consists from high voltage generator and electron source on the basis of vacuum diode or triode schemes. The structure diagram of accelerator is presented in Figure 2,3.

The plasma cathode on the basis of discharge on the surface of dielectrics $[5,6]$ allows top produce the large cross section cathode plasma and forming of electron with large dimensions, see Figure 4.

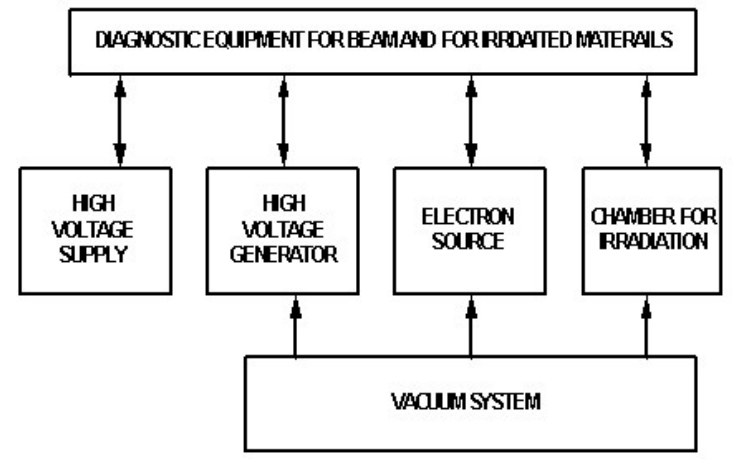

Figure 2.

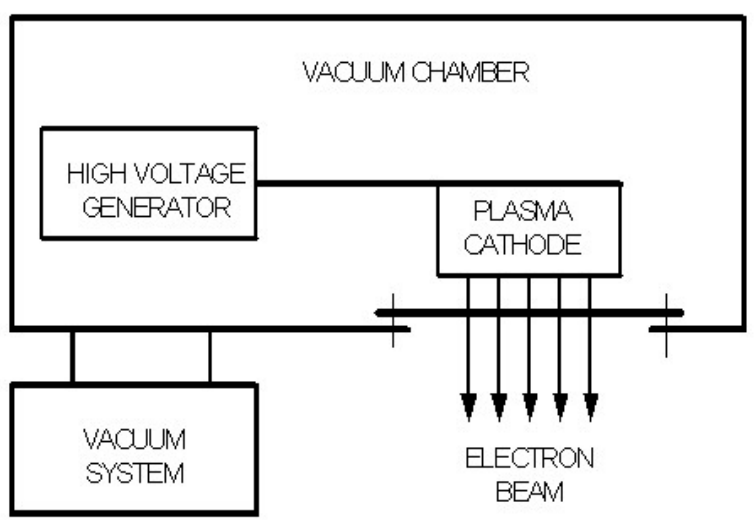

Figure3.

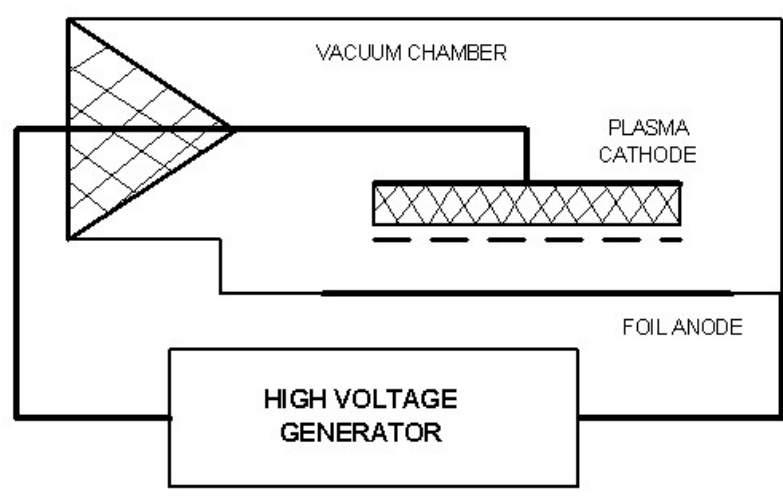

Figure 4.

The triode geometry of electron gun with trigger electrode connected with resistors [7] allows to make regulation of accelerating voltage in $2 \mathrm{D}$ geometry electrode system. Last leads to regulation of current density (power of beam). The general concept of this electron source is given on the Figure 5. 

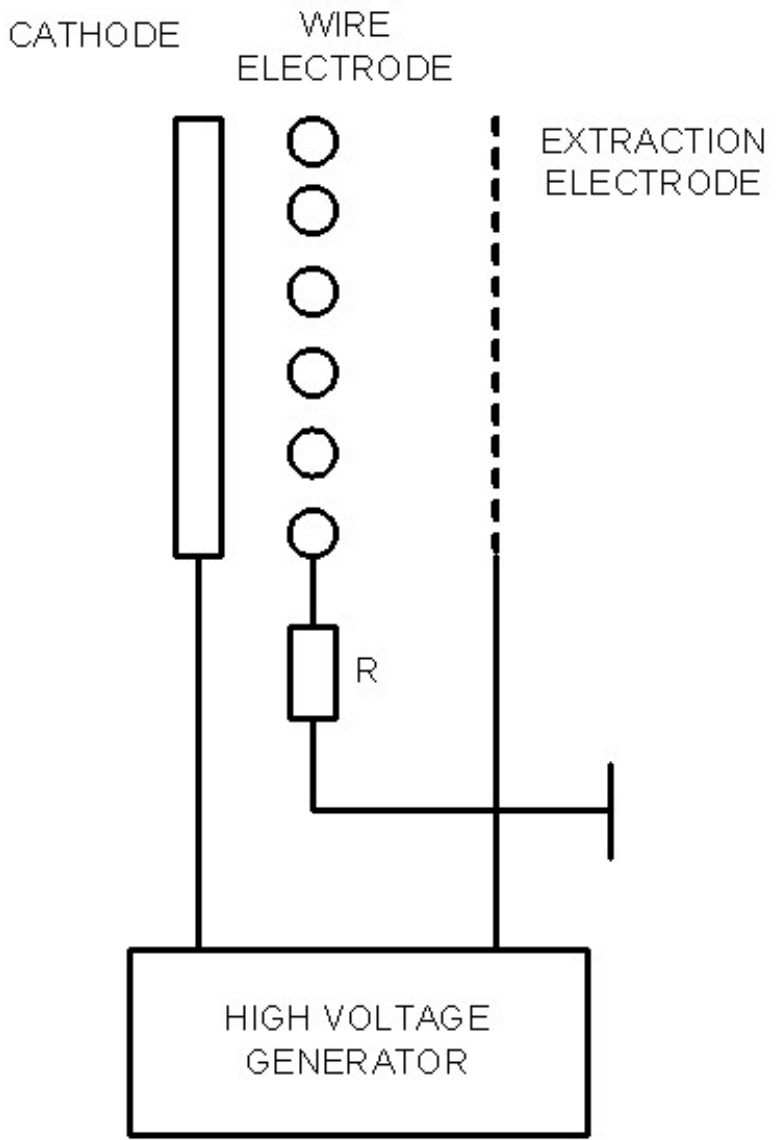

Figure 5.

The regulation of beam power possible by variation of current beam and pulse duration.

Using vacuum insulation for high voltage generator and electron source allows to improving the design of accelerator and increase the lifetime of high voltage elements.

The pulsed accelerator allows to use the pulsed noncontact methods of measurements of beam parameters.

The using of additional thin film's coatings ( carbon for example by CVD) on the output windows from Be ( for example) permits to increase lifetime of foil window [8.9].

The pulsed mode of accelerators leads to simple cooling system in comparison with DC or RF accelerator.

The cost of this accelerator is very low in comparison with DC or RF accelerator.

The operation is very simple. The instrumentation's equipment is reliable for on-lines systems.

The main parameters of accelerators are following:

- $\quad$ kinetic energy

$100-700 \mathrm{keV}$;

- beam current

$100-1000 \mathrm{~A}$;

- pulse duration

$50-1000$ nsec;

- the repetition

$1-50 \mathrm{~Hz}$;

- max dimensions of beam

\section{CONCLUSION}

The concept of pulsed high current electron accelerator with regulations of main parameters allows to consider the development of pulsed accelerator for development for research and industrial applications.

\section{REFERENCE}

[1] R.J. Wood, A.K. Pikaev. Applied Radiation Chemistry: Radiation Processing. A Wiley-Interscience Publication, John Willry \& Sons, Inc. New York, 1994

[2] S. Korenev, Proc. of the XX International Linear Accelerator Conference, 2000, vol. 1, p.645-647

[3] W.L. McLaughlin, A.W. Boyd, K.H. Chaadwick, J.C. McDonald, A, Miller. Dosimetry for Radiation Processing. Taylor\&Francis. London*New York*Philadelphia, 1989

[4] R.V. Latam. High Voltage vacuum insulation: the physical basis. Academic Press. London, 1981.

[5] S.A. Korenev, "The forming of electron beams in the high current diode", Communication of the Joint Institute for Nuclear Research, N 9-81-573, JINR, Dubna, 1981.

[6] 4. Korenev S.A., Rubin N.B. The forming of the bewailed plasma in vacuum prolonged channels. Preprint JINR N 9-82-13, JINR, Dubna, 1982; Journal of Technical Physics, V.53, N 10, 1983, p.1928-1931.

[7] S.A. Korenev. Electron gun for surface treatment of materials, 1989, Patent of Russia No 1501829.

[8] S. Korenev, B. Coll, A. Perry, Surface and Coatings Technology, 1996, V. 86-87, p. 292-301.

[9] S.A. Korenev, "Increasing of life-time of BE foil in the devices for output electron beams to air". Proc. XIV National Conference on accelerators of charge particles. IPHE, Protvino, 1994, p. 144. 